\title{
PROSPECTIVE IS-MST RADAR. POTENTIAL AND DIAGNOSTIC CAPABILITIES
}

\author{
A.P. Potekhin, A.G. Setov, V.P. Lebedev, A.V. Medvedev, D.S. Kushnarev \\ Institute of Solar-Terrestrial Physics SB RAS, Irkutsk, Russia, \\ potekhin@iszf.irk.ru,setov@iszf.irk.ru,lebedev@iszf.irk.ru,medvedev@iszf.irk.ru,ds_k@iszf.irk.ru
}

In the next few years, a new radar is planned to be built near Irkutsk. It should have capabilities of incoherent scatter (IS) radars and mesosphere-stratosphere-troposphere (MST) radars [Zherebtsov et al., 2011]. The IS-MST radar is a phased array of two separated antenna panels with a multichannel digital receiving system, which allows detailed space-time processing of backscattered signal. This paper describes characteristics, configuration, and capabilities of the antenna and transceiver systems of this radar. We estimate its potential in basic operating modes to study the ionosphere by the IS method at heights above $100 \mathrm{~km}$ and the atmosphere with the use of signals scattered from refractive index fluctuations, caused by turbulent mixing at heights below $100 \mathrm{~km}$.

The modeling shows that the radar will allow us to regularly measure neutral atmosphere parameters at heights up to $26 \mathrm{~km}$ as well as to observe mesosphere summer echoes at heights near $85 \mathrm{~km}$ in the presence of charged ice particles (an increase in Schmidt number) and mesosphere winter echoes at heights near $65 \mathrm{~km}$ with increasing background electron density. Evaluation of radar resources at the IS mode in two height ranges 100-600 and 600-2000 km demonstrates that in the daytime and with the accumulation time of $10 \mathrm{~min}$, the upper boundaries of electron density and ionospheric plasma temperature are $\sim 1500$ and $\sim 1300 \mathrm{~km}$ respectively, with the standard deviation of no more than $10 \%$. The upper boundary of plasma drift velocity is $\sim 1100 \mathrm{~km}$ with the standard deviation of $45 \mathrm{~m} / \mathrm{s}$. The estimation of interferometric capabilities of the MST radar shows that it has a high sensitivity to objects of angular size near 7.5 arc min, and its potential accuracy in determining target angles can reach 40 arc sec.

Keywords Incoherent scatter radar $\cdot$ MST radar $\cdot$ Phased array $\cdot$ Atmospheric radar potential $\cdot$ Ionosphere $\cdot$ Atmosphere

\section{INTRODUCTION}

Under the national heliogeophysical complex project, a new radar for extensive atmospheric research is planned to be developed. High radiation power and large aperture of the radar will allow us to study the ionosphere by the incoherent scatter (IS) method and the neutral atmosphere at heights of the mesosphere, stratosphere, and troposphere (MST). The radar is being worked out by Academician A. L. Mints Radiotechnical Institute (RTI) experienced in designing radars. The radar is developed from existing RTI instruments and is modified for atmospheric research purposes. It is necessary to study design features of the IS-MST radar and to assess its scientific potential. 
The main purpose of the radar is to examine the structure and dynamics of the neutral atmosphere and ionosphere, as well as their interactions. Observations of the neutral atmosphere involve measuring the full vector of wind velocity, parameters of turbulence and atmospheric layers at tropospheric, stratospheric, and mesospheric heights. Of special interest are mesospheric echoes [Rapp, Lubken, 2004], which appear in summer and winter, being sometimes accompanied by noctilucent clouds [Romejko et al., 2003]. MST measurements also include studies of meteors at heights of the mesosphere and lower thermosphere, scattering by thermal plasma fluctuations, atmospheric gravity waves, and specular atmospheric layers [Hocking, 2011]. The IS method allows us to obtain ionospheric plasma parameters - electron density, ion and electron temperature, ion content, plasma drift velocity at $100-2000 \mathrm{~km}$ and to observe ionospheric irregularities, coherent echoes, and the D layer. Thus, the radar will enable us to examine physical processes and interactions in the neutral atmosphere-ionosphere-plasmasphere system. The radar is also designed for interferometric observations of the fine structure of atmospheric irregularities and localized targets (satellites, space debris, and meteors), and for passive radioastronomical observations of the Sun and space radio sources. The national heliogeophysical complex includes a number of optical instruments, which will be located near the IS-MST radar and will increase its diagnostic capabilities. For example, observations of the lower and middle atmosphere along with lidar measurements make it possible to explore transfer processes [Bertin et al., 2001].

There are only about ten IS radars and twenty large MST radars in the world. Over the past five years, such instruments as the MST radar MAARSY in Norway [Latteck et al., 2012] and the MST/IS radar PANSY in Antarctica [Sato et al., 2014] have been put into operation. The multifunctional radar EISCAT-3D [McCrea et al., 2015] is planned to be developed. In its technical characteristics, the IS-MST radar should be highly competitive with the state-of-the-art systems. At present, we plan to locate the radar near Lake Baikal. This will enable us to study the atmosphere and ionosphere over the East Siberia, the region that has not had a diagnostic tool with such a large field of view before, and to complement the global radar network. In addition, this will facilitate ecological monitoring of the atmosphere near the lake.

The paper describes the antenna configuration and characteristics of the radar's transceiver system, estimates the height range and the radar potential for working in MST and IS modes and for interferometric and radioastronomical research.

\section{CONFIGURATION AND CHARACTERISTICS OF THE RADAR}

The antenna system of the IS-MST radar consists of two phased arrays (panels) located along the magnetic meridian at a distance of $100 \mathrm{~m}$ from each other and tilted northward and southward at $20^{\circ}$ (Figure 1). Each antenna panel is $40 \times 40$ $\mathrm{m}$ and includes 1536 cross-dipoles in the triangular lattice. The field of view of each antenna panel is $\pm 45^{\circ}$, and the total field of view of the radar is $\pm 65^{\circ}$ from north to south and $\pm 45^{\circ}$ from east to west relative to the magnetic meridian. It should be appreciated that beam steering causes the beam to expand and the gain to decrease; therefore, for this radar configuration the potential increases at a $20^{\circ}$ tilt relative to the horizon and decreases if the beam is directed vertically. The receiving system processes signals of two polarizations. In the radiation mode, the signal has a linear polarization. This facilitates Faraday rotation measurements of the absolute electron density [Shpynev, 2004].

We have chosen the location of the radar to narrow the radio horizon - the distance to nearest obstacles capable of generating clutter. This is required to decrease the minimum distance at which characteristics of a medium can be measured. Residual clutter will be compensated through special signal processing. 
Table 1 lists the main characteristics of the IS-MST radar. Pulse repetition frequency PRF, duty cycle $q$, and pulse length $\tau_{\mathrm{p}}$ depend on chosen operating modes: MST is in the height range 5-100 km, IS-1 in 100-600 km or IS-2 in 600-2000 km. We give these height ranges as an example - the real ones will be specified in accordance with research objectives. For instance, measurements made in the lower and middle atmosphere do not require high pulse repetition frequency because the Doppler bandwidth of MST signals is within $50 \mathrm{~Hz}$; this allows the radar to operate simultaneously at several directions and make additional coherent accumulation of signal to increase the signal-to-noise ratio.

Each antenna panel is partitioned into 12 fragments composed of 128 cross-dipoles and connected to separate transceiver modules. In the module, partial beamforming for signals from 128 antennas occur, thus giving rise to four output receive channels, each with two polarizations and quadratures, available for further processing.

Thus, each antenna array of the radar will form one narrow transmitting beam of $2.5 \times 2.5^{\circ}$ and 12 independent wide receiving beams (12 times as wide). The receiving beams are formed by phase centers spaced apart across antenna panels. The panels are at $100 \mathrm{~m}$ from each other. This makes it possible to combine signals from separate receive channels in order to increase the signal-to-noise ratio, to obtain narrow receiving beams, and to make interferometric observation.
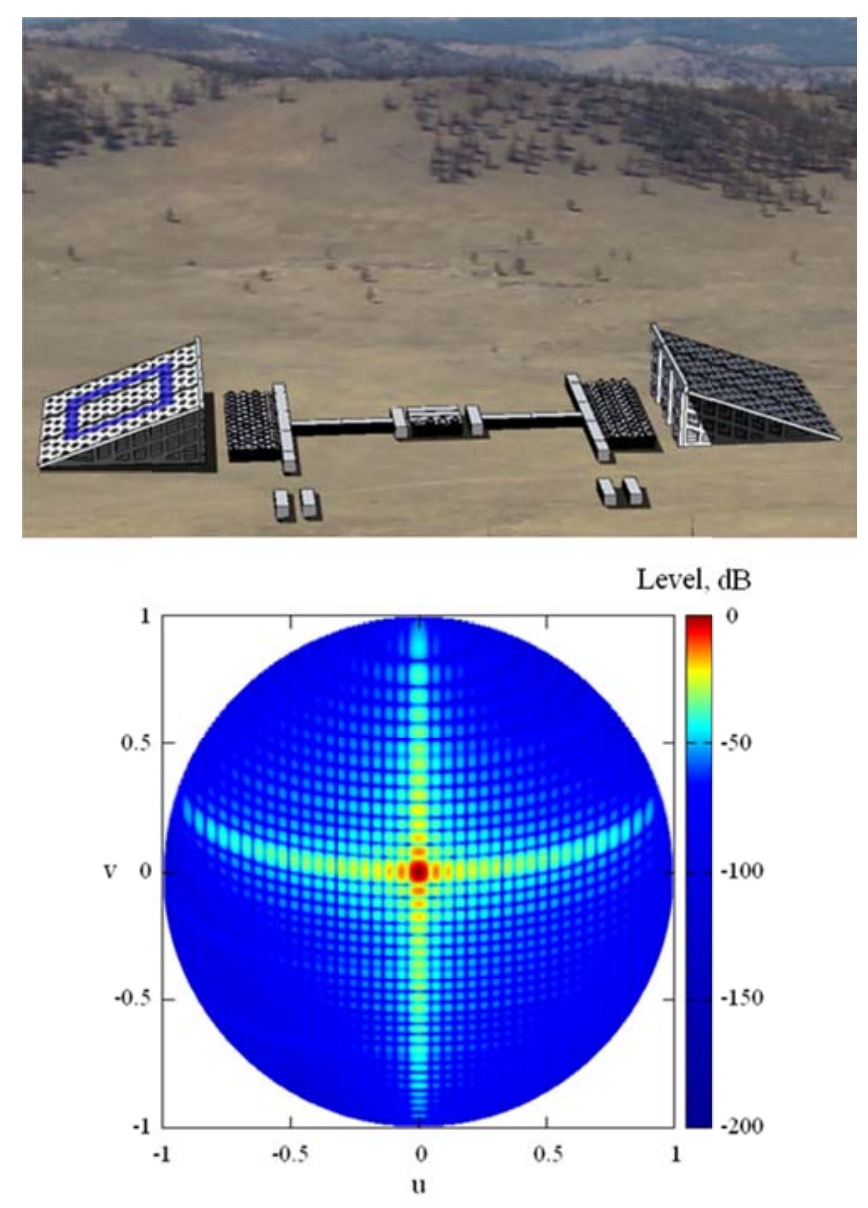

Figure 1. The IS-MST radar (at the top) and array pattern. The array tilt is compensaterd by the beam steering of $20^{\circ}$ for vertical measurements 
Table 1

Characteristics of the IS-MST radar

\begin{tabular}{|c|c|c|}
\hline Characteristic & Notation & Value \\
\hline Frequency range & $f$ & $154-162.7 \mathrm{MHz}$ \\
\hline Configuration & - & $\begin{array}{c}2 \text { square arrays } \\
40 \times 40 \mathrm{~m}\end{array}$ \\
\hline Antenna & - & Cross-dipole \\
\hline $\begin{array}{c}\text { Number } \\
\text { of antennas }\end{array}$ & $N_{t}$ & 3072 \\
\hline Peak power & $P_{t}$ & $2 \mathrm{MW}$ \\
\hline $\begin{array}{c}\text { Effective antenna } \\
\text { area ( } 2 \text { arrays) }\end{array}$ & $A_{\mathrm{e}}$ & $2500 \mathrm{~m}^{2}$ \\
\hline $\begin{array}{c}\text { Mean sidelobe } \\
\text { level }\end{array}$ & - & $-35 \mathrm{~dB}$ \\
\hline $\begin{array}{c}\text { Noise } \\
\text { temperature }\end{array}$ & $T_{\mathrm{e}}$ & $\sim 500 \mathrm{~K}$ \\
\hline Beamwidth & $\theta_{0.5}$ & $\begin{array}{l}2.5 \times 2.5^{\circ} \\
\text { (1 array) }\end{array}$ \\
\hline $\begin{array}{l}\text { Receiver } \\
\text { bandwidth }\end{array}$ & $\Delta B$ & $625 \mathrm{kHz}$ \\
\hline $\begin{array}{l}\text { Bit length of } \\
\text { coded pulse }\end{array}$ & $\tau_{1}$ & $3.2 \mu \mathrm{s}$ \\
\hline $\begin{array}{l}\text { Minimum height } \\
\text { resolution }\end{array}$ & $\Delta r$ & $480 \mathrm{~m}$ \\
\hline $\begin{array}{l}\text { Pulse repetition } \\
\text { frequency } \\
\text { (MST/IS-1/IS-2 } \\
\text { mode) }\end{array}$ & $P R F$ & $1333.3 / 250 / 75 \mathrm{~Hz}$ \\
\hline $\begin{array}{c}\text { Pulse length } \\
\text { (MST/IS-1/IS-2 } \\
\text { mode ) }\end{array}$ & $\tau_{\mathrm{p}}$ & $51.2 / 700 / 6500 \mu \mathrm{s}$ \\
\hline $\begin{array}{c}\text { Duty cycle } \\
\text { ((MST/IS-1/IS-2 } \\
\text { mode })\end{array}$ & $q$ & $6.8 / 17.5 / 37.5 \%$ \\
\hline $\begin{array}{c}\text { Product of mean } \\
\text { power by effective } \\
\text { area }\end{array}$ & $P_{\mathrm{m}} \cdot A_{\mathrm{e}}$ & $\begin{array}{l}3.4 \cdot 10^{8} / 8.75 \cdot 10^{8} / 18.75 \cdot 1 \\
0^{8} \mathrm{~W} \cdot \mathrm{m}^{2}\end{array}$ \\
\hline Field of view & - & $\pm 45^{\circ}$ \\
\hline
\end{tabular}


The signal comes from a digital synthesizer, is transferred to an intermediate frequency, and is converted into the analog form. Then, it divides into all transmitters where it is phased in accordance with the desired direction. The phasing in the transmitting system is accomplished by four-bit analog phase shifters with a phase discrete of $22.5^{\circ}$. The selected phase quantization step of the analog phase shifter results in a gain reduction by $0.06 \mathrm{~dB}$ and in a sidelobe level increase. Synchronizing signals are also formed by a single local oscillator and divide into all transceiver modules; therefore, the radar is phase-coherent with respect to transmitted and received radio pulses.

The receiving system, as opposed to the transmitting one, is all-digital: signals of each polarization from the antennas are intensified in a low noise amplifier, are filtered and digitized. This ensures a wide dynamic range (less than $83 \mathrm{~dB}$ ) that facilitates reception of both weak incoherent signals and strong coherent signals from the neutral and ionized atmosphere. The minimum operating height also depends on the chosen dynamic range because strong clutter leads to saturation in the receiving system, thus impeding their filtering. Received digital signals are split into quadratures, are phased and partially summed up, forming 12 channels from each array in two polarizations and two quadratures, making a total of 96 digital channels. In addition, the transceiver system will enable us to specify arbitrary amplitude and phase distribution in the antenna panel for signals from subarrays of 16 cross-dipoles. Therefore, we can obtain antenna patterns (AP) with a low or zero level of side lobes in selected transmitter and receiver directions.

Figure 1 pictures AP for one antenna panel (1536 elements) in the ground plane. The AP is designed using the classical approach considering the whole antenna field as a single phase center. This allows the calculation time to be reduced. In this case, the effect of mutual coupling between antennas, which causes distortion of AP in individual array elements, is ignored. To compensate tilt of the array, elements of the antenna panel is phased so that the main lobe is directed vertically. The AP has a non-symmetric bend form due to the triangle arrangement of elements and transformation of coordinates to the ground plane. In future, we will simulate the AP more accurately, taking in account fields of individual antennas and interactions between them.

Characteristics of modern IS radars are given, for example, in [Medvedev, 2014]. Most of the radars, constructed before $2000 \mathrm{~s}$, have parabolic antennas with a gain over $40 \mathrm{~dB}$ and $1-5 \mathrm{MW}$ tube transmitters. Some MST radars are outlined in [Hocking, 1997]. In addition to them, we should mention such modern radars as EAR, MAARSY, and PANSY. Unlike IS radars, nearly all MST radars are multibeam phased arrays. This is most likely due to less severe requirements on the power of transmitted signal. The development and cheapening of digital integral devices offer an advantage to phased arrays that enable us to rapidly change beam direction, study several directions at a time, and change the shape of AP at different amplitude and phase distributions. The IS-MST radar with its peak power of $2 \mathrm{MW}$, aperture of $80 \times 40 \mathrm{~m}$, and digital antenna system can make atmospheric observations on a level with first-class instruments. At present, the Institute of Solar-Terrestrial Physics SB RAS studies the ionosphere, using the Irkutsk IS radar [Zherebtsov et al., 2002; Potekhin et al., 2008]. The main shortcomings of the radar are that it cannot make measurements at heights up to 150-200 km and has a fixed pulse-repetition period $(24.4 \mathrm{~Hz})$. The location of the new radar has been chosen to have a narrow radio horizon; hence the radar will not suffer from such shortcomings. The radar is unique also because its two antenna panels are spaced apart by $100 \mathrm{~m}$ and are tilted along the magnetic meridian. This position of the antenna arrays makes it possible to perform interferometric observation and to examine irregularities stretched along the magnetic field. Notice that nowadays there are only two radars working at $158 \mathrm{MHz}$ : Kharkov and Irkutsk radars, which are similarly designed. 


\section{POTENTIAL OF THE RADAR FOR STUDYING THE LOWER, MIDDLE, AND UPPER ATMOSPHERE}

Primary objectives of the radar are to examine dynamic processes in the upper, middle, and lower atmosphere and to study variations in ionospheric plasma parameters and interactions in the atmosphere-ionosphereplasmasphere system. Accordingly, the radar should cover a wide range of heights. Yet medium parameters vary considerably with height, and the atmosphere should be examined by various methods. The main sources of echoes in this range is the incoherent scattering by thermal fluctuations of ionospheric plasma and the Bragg coherent scattering by turbulent fluctuations in the mesosphere-stratosphere-troposphere region, which is sometimes referred to as coherent scattering in the neutral atmosphere.

Operating modes of the IS-MST radar are shown in Figure 2. Separating the radar into two spaced antenna panels quickens scanning of the atmosphere in different directions for high-power backscattered signals (for example, when sounding the height range $100-600 \mathrm{~km}$ in the IS-1 mode). Above $600 \mathrm{~km}$ in the ionosphere (the IS-2 mode), two arrays should work simultaneously in one direction to increase the radar potential. The IS-MST radar has a linear polarization of transmitted signal, which allows us to obtain the ionospheric electron density profile, using Faraday rotation [Shpynev, 2004] or absolute measurements of scattered power.

The MST and IS-1 modes will facilitate the study of several directions with temporal resolution sufficient to determine the full velocity vector of the atmosphere, ionospheric drift, and neutral wind, as well as to examine atmospheric irregularities.

In the lower and middle atmosphere, for each transmitted pulse in each height range, equal to height resolution, we obtain a single complex sample for further processing. Signals at these heights have a coherence time of $\sim 0.1 \mathrm{~s}$, therefore the pulse repetition frequency may be low (tens and hundreds of hertz), but the measurement duration should be long enough to provide necessary frequency resolution (tens of seconds). In general, as the height increases, so does the spectral width of backscattered signal; and signals coming from different pulses lose their coherence. Accordingly, rigorous data-processing requires us to more often obtain samples of signals coming from the same height range (to increase the pulse repetition frequency). Besides, with increasing distance to a region of interest, pulses can be transmitted more rarely to avoid range ambiguities. Yet range resolution should be low, i.e. we should use short pulses or complex pulse-coded signals up to $\sim 100 \mathrm{~km}$, where atmospheric processes have smaller scales. These scales, in turn, depend on the rate of change of atmospheric parameters with height (height scale) and on the extent of atmospheric irregularities and wave disturbances in the plane perpendicular to the radar beam. Thus, at heights of the D and E layers, we should exploit special multipulse transmission modes [Farley, 1972] due to the strict requirements on frequency and range resolution. Starting from 100-200 km, the requirements on pulse width get more relaxed, the signal becomes more incoherent, and for each height range we can take the desired number of samples during one pulse. Problems caused by signal processing can be worsened by conditions of the medium itself. At some heights, intensity of atmospheric disturbances becomes insufficient to obtain echoes and to estimate parameters of the medium. This complicates the study of interactions between different atmospheric layers and makes it dependent on variations in the medium. 

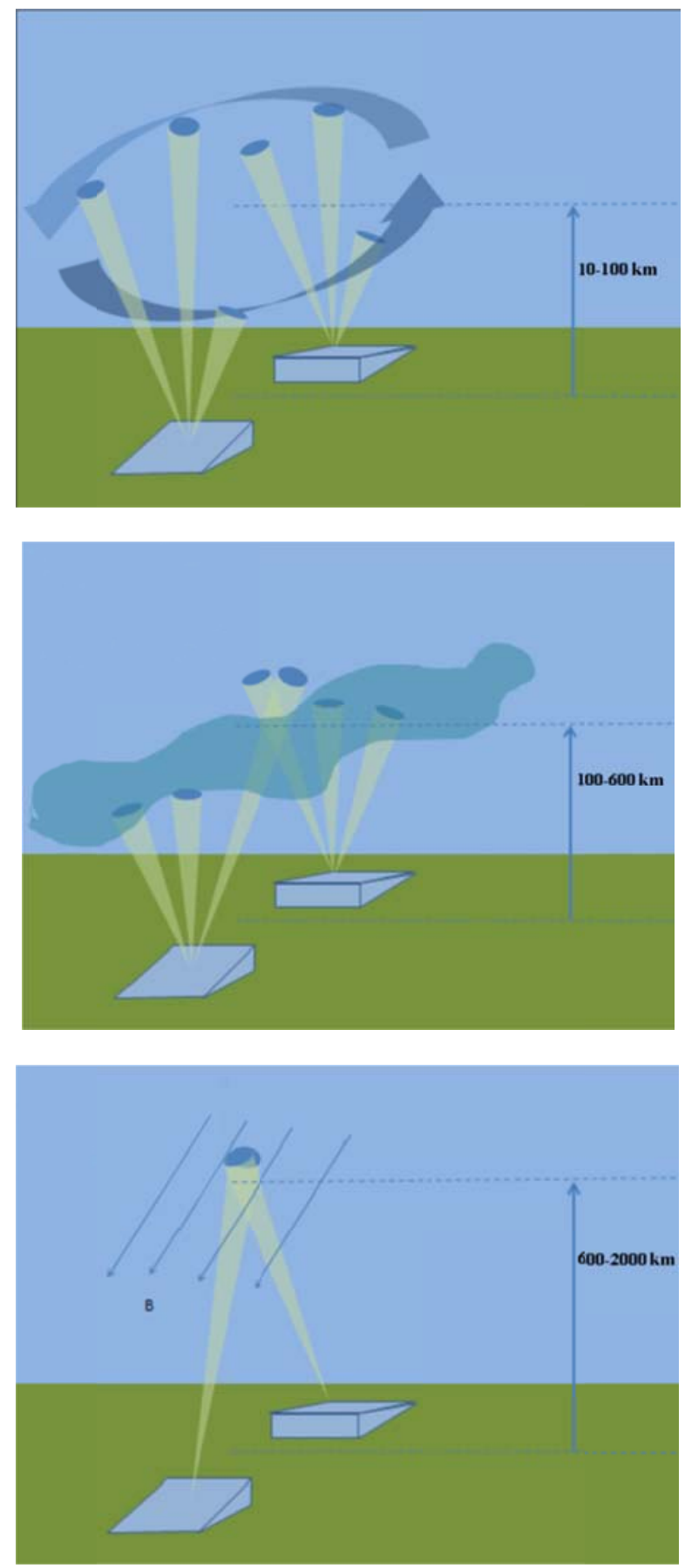

Figure 2. Operating modes of the IS-MST radar. From top to bottom: MST, IS-1, and IS-2 modes

Most MST radars operate at 45-55 MHz [Fukao et al., 1990; Hocking, 1997; Latteck et al., 2012; Sato at el., 2014]. In the 2-30 km range under study, we make regular observations of the troposphere and lower stratosphere, whereas at 60-95 km we observe the mesosphere, including mesospheric echoes. Owing to the decay of low turbulent irregularities in the mesosphere, the scattered-signal power decreases with increasing operating frequency of the radar. This can have an effect on the frequency of observation of mesospheric echoes. Nevertheless, mesospheric echoes, though much weaker, were detected even by radars with the $930 \mathrm{MHz}$ frequency [Rapp et al., 2008]. The frequency range of the IS-MST radar is $\sim 158 \mathrm{MHz}$, hence we need to estimate the frequency dependence of the power of signals scattered by turbulent irregularities. 


\section{Potential assessment technique}

The height range that the radar allows us to study can be estimated by comparing the backscattered signal power with the radar sensitivity. It is more convenient to use volume reflectivity of the unit volume of medium $\sigma_{\mathrm{m}}$ $[1 / \mathrm{m}]$, which depends only on atmospheric parameters. Then, the radar sensitivity is determined by the minimum volume reflectivity $\sigma_{\min }$ the atmosphere should have to obtain the desired statistical significance of estimated medium parameters. If $\sigma_{\mathrm{m}} / \sigma_{\min }>1$, then we can make measurements at this height. The parameter $\sigma_{\min }$ is found from the basic radar equation for the case of volume scattering [Bowles et al., 1962]:

$$
P_{\mathrm{r}}=\frac{P_{\mathrm{t}} L \sigma \tau c \lambda^{2}}{128 \pi^{3} R^{2}} \int_{\theta \phi} G_{\phi}^{2}(\theta, \phi) \sin (\theta) d \theta d \phi
$$

where $P_{\mathrm{t}}$ is the peak radiated power, $L$ are losses in the radar system and during signal propagation though the atmosphere, $\tau$ is the sounding pulse length, $R$ is the distance to the center of the scattering volume, $G(\theta, \varphi)$ is the antenna gain in the direction $(\theta, \varphi)$. To the square of gain $G^{2}$, which accounts for reception and radiation of the same antenna system, corresponds a bidirectional AP $1.8 \times 1.8^{\circ}$ wide for one radar segment. In this case, $\sigma$ determines the fraction of isotropically scattered power per unit scattering volume.

Let us express the assumed power $P_{r}$ through the signal-to-noise ratio $S / N$ :

$$
P_{\mathrm{r}}=k T \Delta B S / N,
$$

where $k$ is the Boltzmann constant, $T$ is the noise temperature of the radar, $\Delta B$ is the frequency bandwidth of the receiver. It is assumed that the receiver has only white thermal noise of the radar and has no clutter (they are said to be eliminated after data processing). The noise characteristics being known, the $S / N$ ratio defines the signal level required to attain the statistical significance of measurements, say, to restrict variance of estimated medium parameters within the prescribed limits.

The integral in (1) accounting for the shape of AP can be approximated by the Gaussian curve for the square aperture array [Hagen, Baumgartner, 1996]:

$$
\iint_{\theta} G_{\phi}^{2}(\theta, \phi) \sin (\theta) d \theta d \phi=0.44 \frac{(4 \pi)^{2} A_{e}}{\lambda^{2}} .
$$

By substituting (2) and (3) in (6), we obtain

$$
\sigma_{\min }=18.2 \frac{\pi R^{2} k T \Delta B S / N}{P_{\mathrm{t}} L \tau c A_{\mathrm{e}} \cos ^{3} \theta}
$$

where $\cos ^{3} \theta$ takes into account losses caused by beam steering ( $\cos \theta$ due to a decrease in aperture projection and $\cos ^{2} \theta$ as an estimate of AP of one cross-dipole). At this stage of development of the IS-MST radar, the losses $L$ are unknown. They are normally clarified during radar calibration. In further calculations, we set $L=0.25$.

In the case of scattering by turbulent irregularities, $\sigma_{\mathrm{m}}$ is calculated from the formula given in [Lübken, 2014]. To estimate atmospheric parameters, we utilize the neutral atmosphere model NRLMSIS-00 and the International Reference Ionosphere model IRI-2012. Turbulence intensity determined by the rate of dissipation of the turbulent energy $\varepsilon$ is taken to be $10^{-3} \mathrm{~m}^{2} \mathrm{~s}^{-3}$ in the troposphere, $10^{-5} \mathrm{~m}^{2} \mathrm{~s}^{-3}$ in the stratosphere, and $10^{-1} \mathrm{~m}^{2} \mathrm{~s}^{-3}$ in the mesosphere; this corresponds to moderate disturbances [Hocking, 1985; Watkins et al., 1988; Kantha, Hocking, 2011; Gavrilov, 2013]. Neutral atmosphere parameters are assumed to be determined from autocorrelation function 
delays. The main measurable parameter in the lower and middle atmosphere is the wind velocity; therefore, the signal-to-noise ratio in (4) is such that the standard deviation of estimated velocity is within $1 \mathrm{~m} / \mathrm{s}$ in the stratosphere and troposphere, and $2 \mathrm{~m} / \mathrm{s}$ in the mesosphere. The expressions for variance of estimated velocity with respect to phase of the autocorrelation function are taken from [Zrnic, 1979].

\section{Potential for tropospheric-stratospheric research}

MST radars regularly measure parameters of signals scattered by turbulent fluctuations of medium [Hocking, 2011]. Turbulent mixing of the neutral component in the stratosphere and troposphere produces the gradient of refractive index. Scattering occurs by refractive index fluctuations with typical scales equal to half the incident wavelength (Bragg's law). The volume reflectivity $\sigma_{\mathrm{m}}$ of refractive index fluctuations caused by turbulent irregularities in the atmosphere depend on the operating frequency of a radar, shape of the spectrum of turbulent fluctuations, and atmospheric parameters [Hocking, 1985]. The turbulence energy spectrum is divided into a buoyancy range, where energy is generated, an inertial range, where the energy slowly decreases, and a viscosity range, where turbulence rapidly decays and the energy is converted into heat. The height of transition between the inertial and viscosity ranges depends on frequency. For example, for VHF radars the transition takes place at heights above $40 \mathrm{~km}$ [Hocking, 1985]. In the middle and upper stratosphere with slowly varying atmospheric parameters and low turbulence intensity, the scattered-signal power decreases. This leads to disappearance of echoes at 30-60 $\mathrm{km}$. An important feature of measurements in the lower and middle atmosphere in a vertical direction is the presence of strong coherent reflections from horizontal extended layers. These echoes have a narrow spectrum less than $1 \mathrm{~Hz}$ wide and aspect sensitivity. In the following, we assess the potential of the radar for scattering only by turbulent fluctuations.

Figure 3 illustrates the ratio $\sigma_{\mathrm{m}} / \sigma_{\min }$ in the troposphere and stratosphere for three operating frequencies: 50 $\mathrm{MHz}$ (the standard frequency of MST radars), $158 \mathrm{MHz}$ (the frequency of the IS-MST radar), and $233 \mathrm{MHz}$ (the frequency of the EISCAT VHF radar). The last frequency has been chosen because the EISCAT VHF radar, which has a relatively low (for IS radars) operating frequency (233 MHz), also performed MST observations [Strelnikova, Rapp, 2010]. Since for the operating mode in the lower atmosphere there are no special requirements on transmission power, Figure 3 also shows the ratio $\sigma_{\mathrm{m}} / \sigma_{\min }$ when only one antenna panel is employed. This allows the temporal resolution to be improved. In the simulation, we have specified the following characteristics: $\tau_{\mathrm{p}}=7 \mu \mathrm{s}$, $P R F=1000 \mathrm{~Hz}$, the accumulation time of $20 \mathrm{~s}$, the Doppler bandwidth of $5 \mathrm{~Hz}$. During the simulation, only frequency of the radar and effective temperature changed, while aperture held its size. It is apparent that when measuring wind velocity in the troposphere and lower stratosphere, a frequency rise enables us to improve potential of the radar (first of all, its temporal resolution) until scattering occurs by turbulent fluctuations in the inertial range. This is due to the fact that the frequency rise causes Doppler shift to increase and thus to be more pronounced against clutter. After transiting to the viscosity range as height rises (it occurs earlier for smaller wavelengths), scattering intensity abruptly decreases. To raise the threshold of wind velocity measurements, we should significantly improve characteristics of the radar and began to use lower frequencies [Hocking, 1997]. Under specified conditions, the IS-MST radar $(158 \mathrm{MHz})$ will facilitate wind velocity measurements up to $\sim 26 \mathrm{~km}$ with a standard deviation within $1 \mathrm{~m} / \mathrm{s}$. 


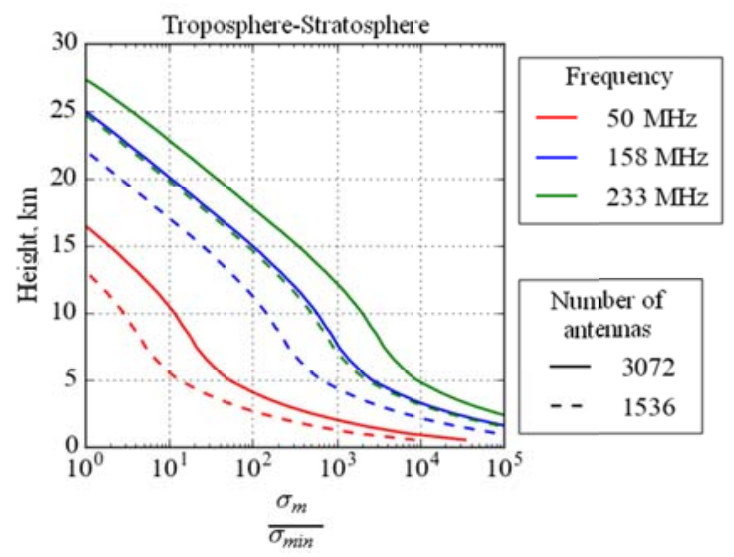

Figure 3. The $\sigma_{\mathrm{m}} / \sigma_{\min }$ ratio in the stratosphere for different frequencies and sizes of the panel in hand

\section{Potential for mesospheric research}

In the mesosphere at the height of the D layer, the signal is further amplified due to enhanced electron density. At the same time, as the height increases, so does the kinematic viscosity of the atmosphere. This causes the decay of small-scale turbulence in the mesosphere and the transition to the viscosity range, and hence loss of scatteredsignal strength [Hocking, 1985].

Nevertheless, at $\sim 85 \mathrm{~km}$ in summer and at $\sim 70 \mathrm{~km}$ in winter, we can observe mesosphere summer echoes (MSE) and mesosphere winter echoes (MWE). The echoes are registered in a wide range from MF to UHF waves [Tereshchenko et al., 2002; Rapp et al., 2008]. Despite their having mostly been detected in the polar region, mesospheric echoes were regularly observed at middle latitudes too. Zeller et al. in [Zeller et al., 2006] show that for a midlatitude radar the frequency of occurrence of echoes is 7 and $0.3 \%$ of the total observation time for MSE and MWE respectively. MST radars also facilitate the study of scattering by meteor trails at $80-100 \mathrm{~km}$, thus allowing measurements of temperature and neutral wind velocity.

In the viscosity range, turbulence decays; however, there exists a mechanism which can reduce scattered signal attenuation and is supposed to produce MSE [Lübken et al., 1998]. In summer at $\sim 85 \mathrm{~km}$, there arise ionized ice particles which decrease electron diffusion. This results in enhancement of electron density fluctuations on small spatial scales [Rapp, Lubken, 2004]. The degree of diffusion attenuation is represented by the Schmidt number Sc that can be as large as several thousands of units [Strelnikov et al., 2009]. MSE at $\sim 82 \mathrm{~km}$ are often accompanied by noctilucent clouds [von Zahn, Bremer, 1999], which are visible with unaided eye [Tereshchenko et al., 2010]. At the height of MWE occurrence $(\sim 70 \mathrm{~km})$, the kinematic viscosity of medium is still sufficiently low, and echo formation does not require ice particles. MWE are thought to appear at high electron density and turbulence of medium. This is confirmed by comprehensive measurements of atmospheric parameters [Lübken et al., 2007].

The previously used formula for finding $\sigma_{\mathrm{m}}$ [Lübken, 2014] allows us to vary Sc. To simulate MSE, we take a layer with increased $S c$ as a necessary condition for echo occurrence. Figure 4 depicts the $\sigma_{\mathrm{m}} / \sigma_{\min }$ ratio for three frequencies and values of Schmidt number. The standard deviation of the estimate for the mesosphere is taken to be $2 \mathrm{~m} / \mathrm{s}$ because observable wind velocities in the mesosphere are higher than those in the troposphere and stratosphere. For the simulation, we have chosen the time of day with high ionospheric electron density because it 
has an effect on the power of echoes [Lübken, 2014]. The Schmidt number increases gradullly from 1 at $82 \mathrm{~km}$ to a specified value at $85 \mathrm{~km}$. Unlike the troposphere and stratosphere, in the mesosphere decay of small-scale turbulence becomes significant, and radars with lower operating frequencies have an advantage regardless of the estimated parameter. It is apparent that the IS-MST radar will enable us to observe MSE at increased Sc. Above 87 $\mathrm{km}$, the decay becomes significant, and the signal strength falls sharply.

MWE occurrence is not followed by an increase in $S c$, but is normally accompanied by enhanced electron density. Figure 5 shows the $\sigma_{\mathrm{m}} / \sigma_{\min }$ ratio for MWE and multiple increase in electron density relative to the value from IRI. The IRI model does not produce values below $65 \mathrm{~km}$. This is illustrated by the figure. The black vertical line depicts the boundary, where the signal is above the threshold of sensitivity of the radar $\left(\sigma_{\mathrm{m}} / \sigma_{\mathrm{min}}=1\right)$. It is shown that a faint echo at $158 \mathrm{MHz}$ may exist if electron density in the D layer increases by an order of magnitude. The frequency of $233 \mathrm{MHz}$ does not allow us to measure the neutral wind velocity in MWE under the specified conditions.

\section{Incoherent scattering}

The IS method is the most informative way of analyzing Earth's upper atmosphere. It facilitates measurements of ionospheric plasma parameters: electron density, ion and electron temperature, plasma drift velocity, ion content, and frequency of collision with the neutral component in the D layer.

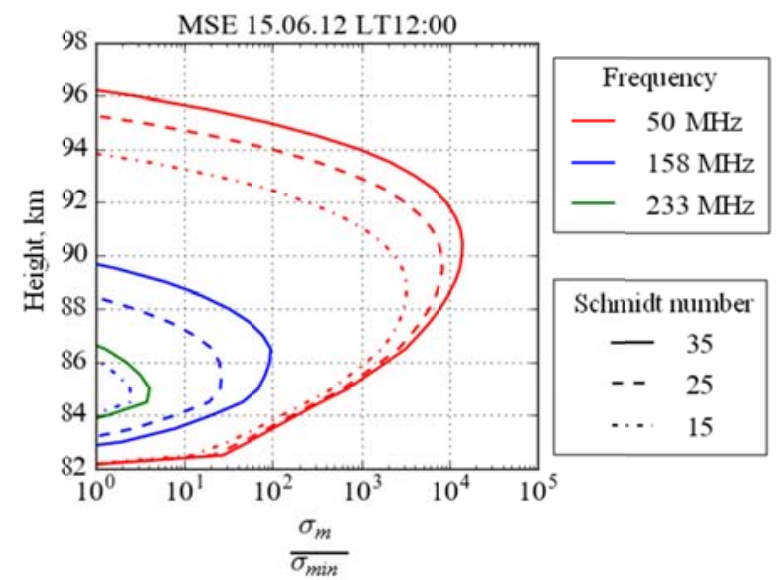

Figure 4. The $\sigma_{\mathrm{m}} / \sigma_{\min }$ ratio for MSE at different frequencies and Schmidt numbers

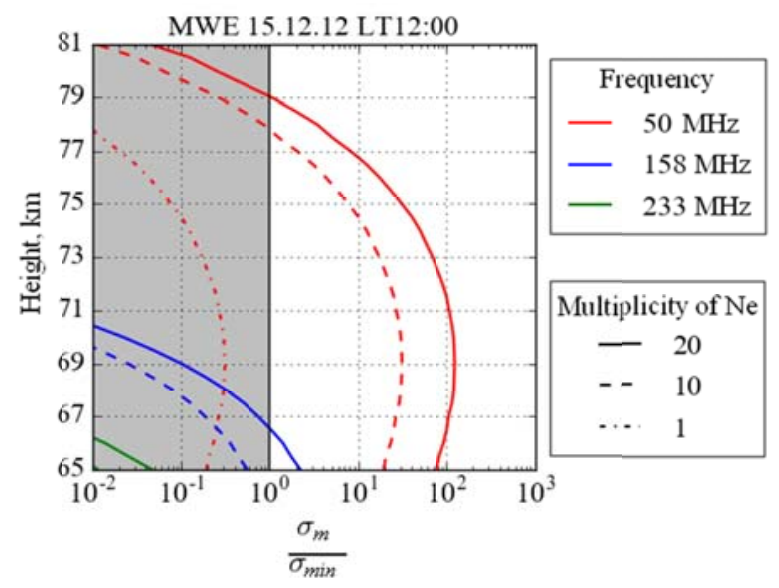

Figure 5. The $\sigma_{\mathrm{m}} / \sigma_{\min }$ ratio for MWE at different frequencies and electron density levels. The vertical line indicates the threshold of sensitivity of the radar 
The volume reflectivity for IS measurements is

$$
\sigma_{\text {IS }}=\frac{N_{\mathrm{e}} \sigma_{\mathrm{e}}}{\left(1+\alpha^{2}\right)\left(1+\alpha^{2}+T_{\mathrm{e}} / T_{\mathrm{i}}\right)},
$$

where $\sigma_{\mathrm{e}} \approx 10^{-28} \mathrm{~m}^{2}$ is the effective cross-section of electron, $\alpha=4 \pi \mathrm{D} / \lambda, D$ is the Debye length, $T_{\mathrm{e}}$ is the electron temperature, $T_{\mathrm{i}}$ is the ion temperature, $N_{\mathrm{e}}$ is the electron density.

We estimate the ionospheric plasma parameters through multiparameter fitting of autocorrelation function or spectrum of a received signal. To analyze variances of the estimated parameters, it is convenient to use the empirical approximation that is given in [Murdin, 1979] for electron density, temperature, and plasma drift velocity. The ionospheric parameters considerably change with height. This has a marked effect on backscattered-signal spectrum and typical scales of current processes (height scale, tidal wave length). Accordingly, the operating mode of the radar should be changed for different heights. The radar has two basic operating modes for ionospheric observations at heights from $100 \mathrm{~km}$ : IS-1 and IS-2 (see Figure 2). The former mode is meant for heights $100-600 \mathrm{~km}$ and is characterized by the following parameters: the pulse length $\tau_{\mathrm{p}}=700 \mu \mathrm{s}, P R F=250 \mathrm{~Hz}$. Besides, the IS- 1 mode suggests using only one radar segment. In the IS- 2 mode for heights from $600 \mathrm{~km}$, we employ longer pulses $\tau_{\mathrm{p}}=6500$ $\mu \mathrm{s}$, and frequency of their repetition decreases to $P R F=75 \mathrm{~Hz}$.

Figure 6 pictures the $\sigma_{\mathrm{m}} / \sigma_{\mathrm{min}}$ ratio for the IS- 1 and IS-2 modes for estimated electron density and temperature with a standard deviation within $10 \%$ and plasma drift velocity with a standard deviation of no more than $45 \mathrm{~m} / \mathrm{s}$. The accumulation time is assumed to be $10 \mathrm{~min}$. The modeling is made for summer noon when electron density is sufficiently high. It is apparent that the plasma drift velocity, even with a high statistical deviation, cannot be measured at a lower height than electron density and temperature. At the height of operating mode change $(600 \mathrm{~km})$, the pulse length increases, but the pulse repetition frequency decreases; therefore, the $\sigma_{\mathrm{m}} / \sigma_{\min }$ ratio rises slightly. Above $600 \mathrm{~km}, \sigma_{\mathrm{m}} / \sigma_{\min }$ is low even in the maximum operating mode; hence, if the real potential of the radar turns out to be lower due to neglected losses in transmitter/receiver or processing system, the threshold of determining plasma parameters may lower to $800-1000 \mathrm{~km}$. Nevertheless, under disturbed conditions the accumulation time may become shorter, and thus temporal resolution may be improved. This allows a detailed study of ionospheric processes.

To comparatively assess the potential, Figure 7 shows the $\sigma_{\mathrm{m}} / \sigma_{\min }$ ratio, found by the same method for the Irkutsk Incoherent Scatter Radar (IISR), which has been used for ionospheric research for over ten years [Zherebtsov et al., 2002; Medvedev, 2014]. The IISR has a frequency scanning principle and a smaller effective antenna area than the IS-MST radar has. It receives backscattered signals of only one polarization and transmits pulses no longer than $900 \mu$ s with a pulse repetition frequency of $24.4 \mathrm{~Hz}$. These limitations hamper the comprehensive study of the ionosphere at heights above $600 \mathrm{~km}$. Moreover, clutter restricts the lower range of the heights of interest, thus making it impossible to observe the ionosphere and atmosphere at heights up to $100 \mathrm{~km}$. The boundary of velocity measurements is seen to be situated much lower than that shown in Figure 6. This is due to the low pulse repetition frequency of IISR and consequently due to the short sample length for the same accumulation time (10 min). 

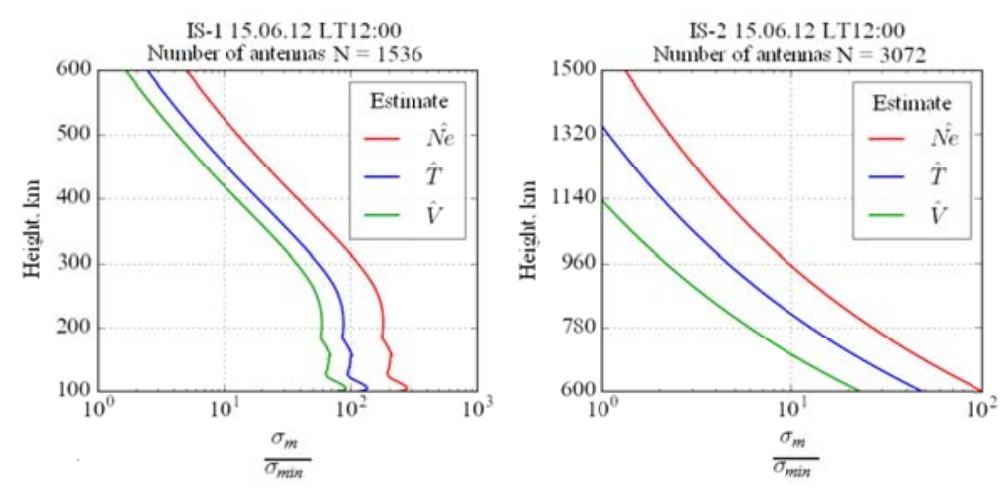

Figure 6. The $\sigma_{\mathrm{m}} / \sigma_{\min }$ ratio for estimated electron density, temperature, and plasma drift velocity. The number of antennas in the IS-1 and IS-2 modes determines the functioning of the radar using one (1536 antennas) or two (3072 antennas) segments respectively.

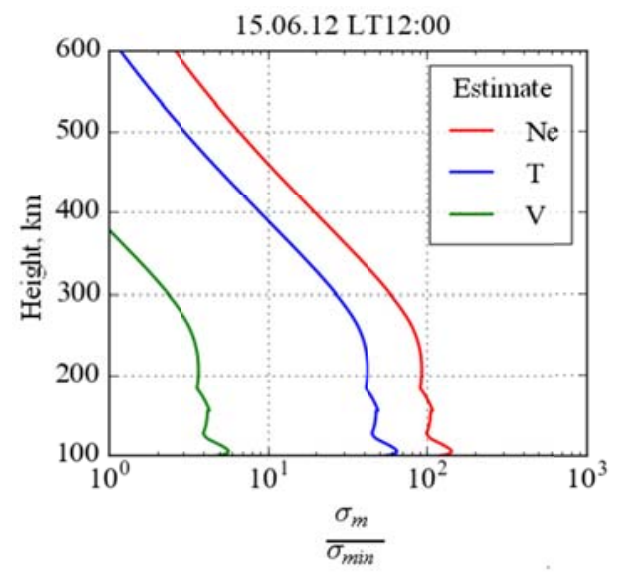

Figure 7. The $\sigma_{\mathrm{m}} / \sigma_{\min }$ ratio for the IISR

When comparing Figures 6 and 7, we should take into account that the plot in Figure 6 (the IS-1 mode, the upper panel) is constructed for one array of the IS-MST radar whereas two arrays enable us to analyze two directions at a time with the same power consumption. However, we should note that by contrast to the IISR, digital processing of signals in each antenna of the IS-MST radar increases power consumption. This is most noticeable when the radar works in the passive operating mode.

\section{ESTIMATED SENSITIVITY OF THE RADAR IN STUDYING COHERENCE PROPERTIES OF SCATTERED SIGNALS}

At present, interferometric methods offer the greatest promise for studying the lower, middle, and upper atmosphere. They have been extensively developed both theoretically and experimentally. Image radars which enable reconstruction of the fine structure of irregularities in a scattering volume from radar measurements are being worked out. Such a method is employed to study, for example, turbulence and neutral wind velocity in the lower atmosphere. Radar images of irregularities provide further insight into physical mechanisms responsible for their formation.

The main target of research in this method, associated with parameters of a medium, is the standardized correlation coefficient (CC) of signals $u_{1}(t)$ and $u_{2}(t)$, received by two and more spaced antennas: 


$$
\eta=\left\langle u_{1} u_{2}^{*}\right\rangle / \sqrt{\left\langle\left|u_{1}\right|^{2}\right\rangle\left\langle\left|u_{2}\right|^{2}\right\rangle} .
$$

Nowadays, the interferometric method is the most advanced in the study of such objects as spacecraft, space debris, meteors, meteor trails, backscattered signals from ionospheric irregularities, turbulent structures stretched along the magnetic field, artificial heating region, turbulent irregularities in the mesosphere and lower atmosphere, internal gravity waves. Of great practical interest is the study of cosmic radio emission, particularly the distribution of radio emission over the solar disk and its dynamics.

Specificity of the antenna system of the IS-MST radar allows us to consider it as a two-position interferometer with the base $D=140 \mathrm{~m}$; its transmitting and receiving antennas are identical and have a half-power beamwidth of $2.5^{\circ}$. The main lobe of AP can be approximated by the Gaussian curve with an accuracy of $5 \%$ :

$$
F_{\text {beam }}(\theta)=\exp \left(-\frac{\theta^{2}}{2 \sigma_{\text {beam }}^{2}}\right)
$$

where $\sigma_{\text {beam }}$ is the effective beamwidth.

Sensitivity of the IS-MST radar in observation of ionospheric irregularities and radioastronomical objects can be assessed as follows. Let us consider an observed object of Gaussian form:

$$
f(\theta)=\left\{\begin{array}{c}
\left\langle|\Delta n(\theta)|^{2}\right\rangle \\
W(\theta)
\end{array}=A \exp \left(-\frac{\left(\theta-\theta_{0}\right)^{2}}{2 \sigma_{\theta}^{2}}\right),\right.
$$

where $\left\langle|\Delta n(\theta)|^{2}\right\rangle$ is the electron density disturbance $\left(\sigma_{\text {beam }}=0.711\right), W(\theta)$ is the average power flux $\left(\sigma_{\text {beam }}=1.422\right), \sigma_{\theta}$ is the characteristic angular size of the object, $\theta_{0}$ is the object's center of mass. Although we study different objects (electron density disturbances and distributed radio source), the resulting equation for $\mathrm{CC}$ takes the form

$$
\eta=\exp (i \theta_{0} \underbrace{\frac{k_{a} \sigma_{\text {beam }}^{2}}{\sigma_{\text {beam }}^{2}+2 \sigma_{\theta}^{2}}}_{\mathrm{k}_{\mathrm{ef}}}) \underbrace{\exp \left(-\frac{k_{a}^{2} \sigma_{\text {beam }}^{2} \sigma_{\theta}^{2}}{2\left(\sigma_{\text {beam }}^{2}+2 \sigma_{\theta}^{2}\right)}\right)}_{\text {CC amplitude }},
$$

where $k_{a}=2 \pi \mathrm{D} / \lambda=463.28$ (el. degree/( 1 degree of elevation angle ), $\lambda=1.9 \mathrm{~m}$ is the wavelength.

Figure 8 illustrates the dependence of CC amplitude and effective slope of CC phase-angle response $k_{\mathrm{ef}}$ on the angular size of the object $\sigma_{\theta}$. This figure indicates that as angular sizes of the object increase, the slope of phaseangle response becomes steeper, i.e., the interferometer becomes less sensitive to change of the angular position of the observed object. 

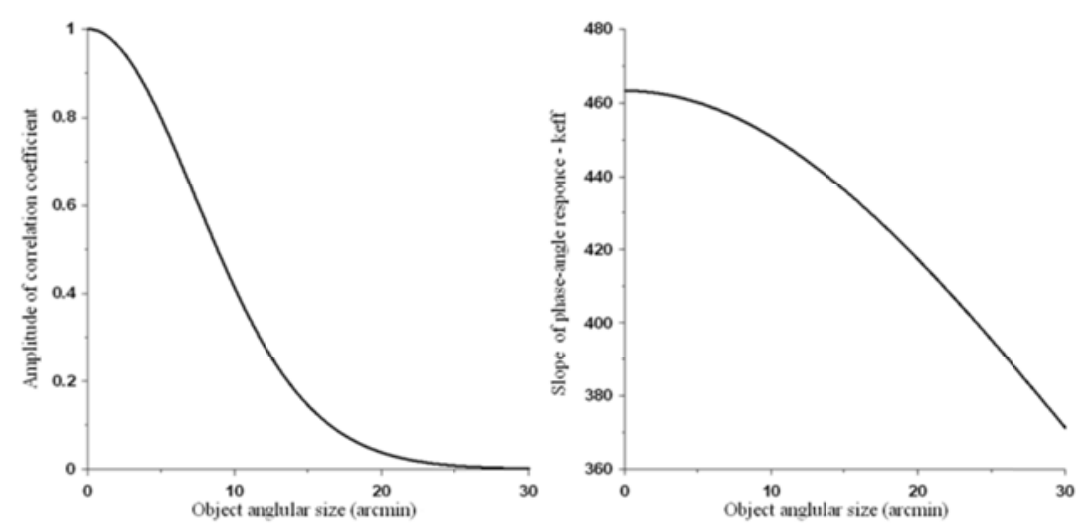

Figure 8. The CC amplitude (on the left) and the slope of phase-angle response as a function of angular size of the observed object $\sigma_{\theta}$

The maximum derivative of the CC amplitude corresponds to an object with an angular size of 7.3 arcmin, i.e., in this region $\mathrm{CC}$ is most sensitive to changes of angular sizes of the object. This enables us to use $\mathrm{CC}$ to determine transverse sizes of the observed object. The high sensitivity of the IS-MST radar to angular sizes of object of about 7.5 arcmin offers great promise for studying the fine structure of irregularities in the lower, middle, and upper atmosphere, as well as the fine structure of solar radio emission.

Potential capabilities of the IS-MST radar in scanning spacecraft and space debris

We can express the cross-section of spacecraft through the basic radar equation:

$$
\sigma=\frac{S}{N} \frac{(4 \pi)^{3} R^{4} k T \Delta B}{P G^{2} \lambda^{2}}
$$

Since the greatest danger arises from small-sized space debris, let us assess sensitivity of the IS-MST radar for objects in the Rayleigh region, i.e., at $k_{0} a<0.75$ ( $k_{0}$ is the wave number, $a$ is the radius of metal sphere). In the region of Rayleigh scattering, for the metal sphere the ratio between its diameter $d$ and cross-section has the following dependence:

$$
\sigma=9\left(\frac{\pi d}{\lambda}\right)^{4} \pi\left(\frac{d}{2}\right)^{2}
$$

Table 2 lists calculated sizes of debris for $S / N=10$.

Table 2

Calculated particle sizes at $S / N=10$

\begin{tabular}{|c|c|c|c|c|c|}
\hline$R, \mathrm{~km}$ & 100 & 400 & 1000 & 1500 & 2000 \\
\hline$d, \mathrm{~cm}$ & 0.7 & 17.5 & 32.2 & 42.3 & 51.2 \\
\hline
\end{tabular}

Figure 9 shows the sensitivity of the IS-MST radar in scanning spacecraft and space debris for different signal-tonoise ratios. ISTP SB RAS has mastered techniques and algorithms for the IISR, which ensure stable operation of the radar at $S / N<1$. 


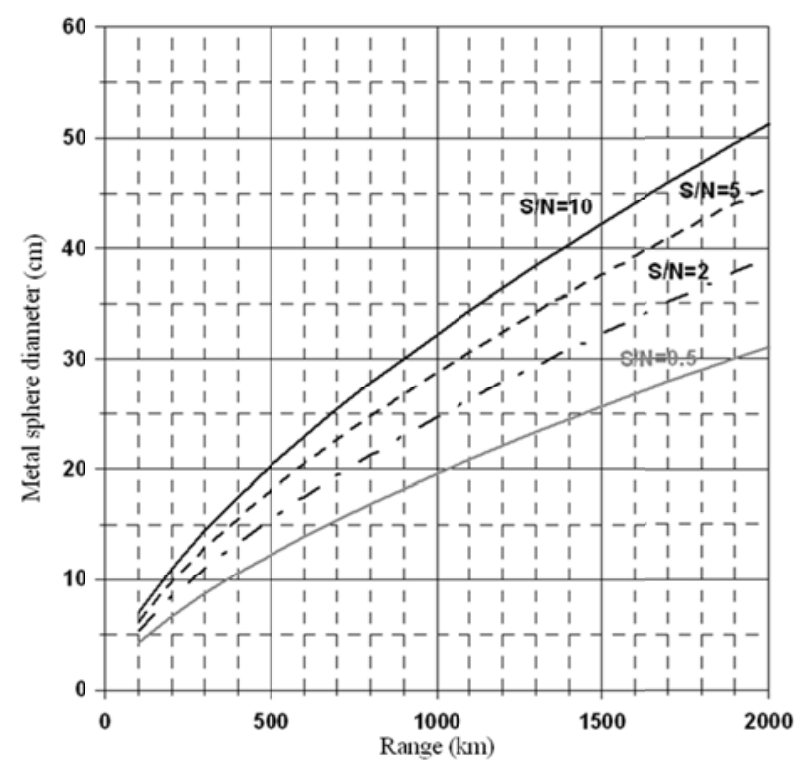

Figure 9. Sensitivity of the IS-MST radar in scanning spacecraft and space debris for different signal-to-noise ratios

At present, we have achieved the following positioning accuracy: the standard deviation in range is $\sim 100-300$ $\mathrm{m}$, the standard error in determining target angles is $\sim 2-5$ arcmin. The techniques worked out for the IISR can be applied to the IS-MST radar, with potential accuracy in determining angles reaching 40 arcsec.

The wide field of view of the IS-MST radar equal to $90^{\circ}$ will allow us to observe spacecraft and space debris for more than $100 \mathrm{~s}$. This suffices to determine orbital parameters with accuracy necessary to predict the next circuit of the object. Thus, IS-MST radar data suffice to define orbital parameters of small-sized space debris.

\section{CONCLUSION}

The IS-MST radar is designed for complex diagnostics of the atmosphere from the troposphere to the plasmasphere; therefore, the range of heights under study should be as broad as possible. Atmosphere-radiosounding methods offer the possibility to continuously examine turbulence in the troposphere, stratosphere, and mesosphere, to observe meteor echo in the mesosphere and lower thermosphere, and to study the ionosphere at heights from $60 \mathrm{~km}$. Almost all types of radar echo allow us to obtain a 3D velocity vector to explore the atmospheric dynamics and processes of transfer between atmospheric layers.

Most modern radars used to study Earth's atmosphere have phased antenna arrays. This type of antenna system as opposed to parabolic dishes enables us to change beam direction without any mechanical motion of the antenna system. The fast progress in digital systems facilitates the accumulation of signals from separate structural elements of antenna panel for further processing, in particular for interferometric observation. The prospective IS-MST radar also represents a phased antenna system with 24 receive channels, each with complex amplitude backscattered signals of two polarizations. The chosen position of its antenna arrays provides a wide field of view for the radar northward and southward along the magnetic meridian. The new instrument has characteristics comparable to characteristics of state-of-the-art IS and MST radars. 
Spacing of antenna arrays gives an interferometric base of $140 \mathrm{~m}$, thus enabling us to examine the fine structure of atmospheric irregularities. We have shown that the antenna system offers the possibility to use the correlation coefficient to determine orbital parameters of spacecraft and space debris.

We have estimated the height range of the IS-MST radar, which represents the total potential of the radar for atmospheric research. We have demonstrated its capabilities for regular measurements of turbulent irregularities in the troposphere and stratosphere. We have found that in the lower and middle atmosphere the efficiency of measurements of wind velocity is higher for radars with higher frequency if scattering occurs in an inertial range of spectrum of turbulent refractive-index fluctuations. The lower boundary of the height range in the troposphere will depend on signal processing system, specifically on filtering of local clutter. We have shown that the radar enables us to observe echoes in the summer mesosphere, which are scattered by turbulent fluctuations in the presence of charged ice particles, which corresponds to an increase in the Schmidt number. It also facilitates observation of rarer winter mesospheric echoes [Zeller et al., 2006] under enhanced electron density. In the mesosphere, MST radars with a frequency of $50 \mathrm{MHz}$ [Hocking, 1997] have an advantage over the IS-MST radar because turbulent fluctuations with typical scales equal to half the wavelength begin to decay with increasing height. The IS-MST radar enables us to measure plasma parameters - electron density, temperature, and plasma drift velocity - through multiparameter fitting in the IS-1 and IS-2 modes (height ranges 100-600 and 600-1500 km respectively) with sufficient statistical accuracy. Comparative modeling of potential of the IISR has revealed that the new instrument has great diagnostic capabilities of studying the ionosphere at heights from $400 \mathrm{~km}$, and allows us to explore the atmosphere below $100 \mathrm{~km}$.

The work was funded by the RF President's Grant for Support of Leading Scientific Schools No. NSh6894.2016.5.

\section{REFERENCES}

Bertin F., Campistron B., Caccia J.L., Wilson R. Mixing processes in a tropopause folding observed by a network of ST radar and lidar. Ann. Geophys. 2001, vol. 19, pp. 953-963.

Bowles K.L., Ochs G.R., Green J.L. On the absolute intensity of incoherent scatter echoes from the ionosphere. J. Res. of the National Bureau of Standards-D. Radio Propagation. 1962, vol. 66D, no. 4, pp. 395-407.

Farley D.T. Multiple-pulse incoherent-scatter correlation function measurements. Radio Sci. 1972, vol. 7, no. 6, pp. 661-666. DOI: 10.1029/RS007i006p00661.

Fukao S., Sato T., Tsuda T., et al. MU radar: New capabilities and system calibrations. Radio Sci. 1990, vol. 25, no. 4, pp. 477-485. DOI: 10.1029/RS025i004p00477.

Gavrilov N.M. Estimates of turbulent diffusivities and energy dissipation rates from satellite measurements of spectra of stratospheric refractivity perturbations. Atm. Chem. Phys. 2013, vol. 13, pp. 12107-12116. DOI: 10.5194/acp-1312107-2013.

Hagen J.B., Baumgartner H.A. Backscatter gain of aperture antennas. Radio Sci. 1996, vol. 31, no. 4, pp. $693-$ 699. DOI: $10.1029 / 96 \mathrm{RS} 01095$.

Hocking W.K. Measurement of turbulent energy dissipation rates in the middle atmosphere by radar techniques: A review. Radio Sci. 1985, vol. 20, no. 6, pp. 1403-1422. DOI: 10.1029/RS020i006p01403. 
Hocking W.K. Recent advances in radar instrumentation and techniques for studies of the mesosphere, stratosphere and troposphere. Radio Sci. 1997, vol. 32, no. 6, pp. 2241-2270. DOI: 10.1029/97RS02781.

Hocking W.K. A review of mesosphere-stratosphere-troposphere (MST) radar developments and studies, circa 1997-2008. J. Atm. and Solar-Terr. Phys. 2011, vol. 73, pp. 848-882. DOI: 10.1016/j.jastp.2010.12.009.

Kantha L., Hocking W. K. Dissipation rates of turbulence kinetic energy in the free atmosphere: MST radar and radiosondes. J. Atm. and Solar-Terr. Phys. 2011, vol. 73, pp. 1043-1051. DOI: 10.1016/j.jastp.2010.11.024.

Latteck R., Singer W., Rapp M., et al. MAARSY: The new MST radar on Andøya - System description and first results. Radio Sci. 2012, vol. 47, RS1006. DOI: 10.1029/2011RS004775.

Lübken F.-J. Turbulent scattering for radars: A summary. J. Atm. and Solar-Terr. Phys. 2014, vol. 107, pp. 1-7. DOI: 10.1016/j.jastp.2013.10.015.

Lübken F.-J., Rapp M., Blix T., Thrane E. Microphysical and turbulent measurements of the Schmidt number in the vicinity of polar mesosphere summer echoes. Geophys. Res. Lett. 1998, vol. 25, no. 6, pp. 893-896.

Lübken F.-J., Singer W., Latteck R., Strelnikova I. Radar measurements of turbulence, electron densities, and absolute reflectivities during polar mesosphere winter echoes (PMWE). Adv. Space Res. 2007, vol. 40, pp. $758-764$. DOI: $10.1016 /$ j.asr.2007.01.015.

Medvedev A.V. Razvitie metodov i apparatnykh sredstv radiofizicheskikh issledovanii verkhnei atmosfery Zemlina Irkutskom radare nekogerentnogo rasseyaniya [Development of methods and hardware of radiophysical investigations of Earth's upper atmosphere at the Irkutsk Incoherent Scatter Radar]. Dr. Phys. and Math. Sci. Diss. Irkutsk, 2014. 225 p. (In Russian).

McCrea I., Aikio A., Alfonsi L., et al. The science case for the EISCAT_3D radar. Progress in Earth and Planetary Science. 2015, vol. 2. DOI: 10.1186/s40645-015-0051-8.

Murdin J. Errors in incoherent scatter radar measurements. EISCAT Technical Note. 1979, no. 79/16, 40 p.

Potekhin A.P., Medvedev A.V., Zavorin A.V., et al. Recording and control digital systems of the Irkutsk Incoherent Scatter Radar. Solnechno-zemnaya fizika [Solar-Terrestrial Physics]. 2008, iss. 11, pp. 77-86. (In Russian).

Rapp M., Lübken F.-J. Polar mesosphere summer echoes (PMSE): Review of observations and current understanding. Atmos. Chem. Phys. 2004, vol. 4, pp. 2601-2633. DOI: 10.5194/ acp-4-2601-2004.

Rapp M., Strelnikova I., Latteck R., et al. Polar mesosphere summer echoes (PMSE) studied at Bragg wavelength of $2.8 \mathrm{~m}, 67 \mathrm{~cm}$, and $16 \mathrm{~cm}$. J. Atm. and Solar-Terr. Phys. 2008, vol. 70, pp. 947-961. DOI: 10.1016/j.jastp.2007.11.005.

Romejko V.A., Dalin P.A., Pertsev N.N. Forty years of noctilucent cloud observations near Moscow: Database and simple statistics. J. Geophys. Res. 2003, vol. 108, no. D8, pp. PMR 10-1-10-6. DOI: 10.1029/2002JD002364.

Sato K., Tsutsumi M., Sato T., et al. Program of the Antarctic Syowa MST/IS radar (PANSY). J. Atm. and SolarTerr. Phys. 2014, vol. 118, pp. 2-15. DOI: 10.1016/j.jastp.2013.08.022.

Shpynev B.G. Incoherent scatter Faraday rotation measurements on a radar with single linear polarization. Radio Sci. 2004, vol. 39, RS3001. DOI: 10.1029/2001RS002523.

Strelnikov B., Rapp M., Strelnikova I., et al. Small-scale structures in neutrals and charged aerosol particles as observed during the ECOMA/MASS rocket campaign. Ann. Geophys. 2009, vol. 27, pp. 1449-1456. DOI: 10.5194/angeo-27-1449-2009. 
Strelnikova I., Rapp M. Studies of polar mesosphere summer echoes with the EISCAT VHF and UHF radars: Information contained in the spectral shape. Adv. Space Res. 2010, vol. 45, pp. 247-259. DOI: 10.1016/j.asr.2009.09.007.

Tereshchenko V.A., Tereshchenko V.D., Chernyakov S.M. Winter polar stratospheric clouds at high latitudes in 2010. Vestnik MGTU [Bulletin of Moscow State Technical University]. 2010, vol. 13, no. 4/2, pp. 1052-1059. (In Russian).

Tereshchenko V.D., Tereshchenko V.A., Kovalevich T.V. Seasonal variations of polar mesospheric echo of medium radio waves. Proc. $20^{\text {th }}$ National Conference on Radio Wave Propagation. Nizhny Novgorod, July 2-4, 2002. Nizhny Novgorod: NIRFI, 2002, pp. 135-136. (In Russian).

Von Zahn U., Bremer J. Simultaneous and common-volume observations of noctilucent clouds and polar mesosphere summer echoes. Geophys. Res. Lett. 1999, vol. 26, no. 11, pp. 1521-1524.

Watkins B.J., Philbrick C.R., Balsley B.B. Turbulence energy dissipation rates and inner scale sizes from rocket and radar data. J. Geophys. Res. 1988, vol. 93, no. D6, pp. 7009-7014.

Zeller O., Zecha M., Bremer J., et al. Mean characteristics of mesosphere winter echoes at mid- and high latitudes. J. Atm. and Solar-Terr. Phys. 2006, vol. 68, pp. 1087-1104. DOI: 10.1016/j.jastp.2006.02.015.

Zherebtsov G.A., Zavorin A.V., Medvedev A.V., et al. The Irkutsk Incoherent Scatter Radar. Radiotekhnika i electronika [J. of Communications Technology and Electronics]. 2002, vol. 47, no. 11, pp. 1339-1345. (In Russian).

Zherebtsov G.A., Potekhin A.P., Kurkin V.I., et al. Radiophysical and optical instruments of the National Heliogeophysical Complex of RAS. Radiowave Propagation. Proc. XXIII National Scientific Conference. YoshkarOla, May 23-26, 2011. Yoshkar-Ola: MarGTU, 2011, vol. 1, pp. 47-54. (In Russian).

Zrnic D. Estimation of spectral moments for weather echoes. IEEE Trans. Geoscience Electronics. 1979, vol. GE-17, no. 4, pp. 113-128. DOI: 10.1109/TGE.1979.294638. 